\title{
Preface of the special issue for the Oberwolfach Workshop on Computability Theory 2018
}

\author{
Vasco Brattka \\ Faculty of Computer Science, Universität der Bundeswehr München, Neubiberg, Germany \\ Department of Mathematics and Applied Mathematics, University of Cape Town, Rondebosch, South Africa \\ Vasco.Brattka@cca-net.de \\ Rod Downey \\ School of Mathematics and Computer Sciences, Victoria University, Wellington, New Zealand \\ Rod. Downey@msor . vuw.ac.nz \\ Julia F. Knight \\ Department of Mathematics, University of Notre Dame, Notre Dame, IN, USA \\ knight.1end.edu

\section{Steffen Lempp} \\ Department of Mathematics, University of Wisconsin, Madison, WI, USA \\ lempp@math.wisc.edu
}

This double issue of the journal Computability is a special issue dedicated to the Oberwolfach Workshop on Computability Theory that took place in January 2018 at the international mathematics research institute in Oberwolfach, Germany [1]. The workshop has covered computability theory in its entire breadth, including modern applications.

Computability and computable enumerability are two of the fundamental notions of mathematics. Interest in effectiveness is already apparent in the famous Hilbert problems, in particular the second and tenth, and in early 20th century work of Dehn, initiating the study of word problems in group theory. The last decade has seen both completely new subareas develop as well as remarkable growth in two-way interactions between classical computability theory and areas of applications. There is also a great deal of work on algorithmic randomness, reverse mathematics, computable analysis, and in computable structure theory/computable model theory. The goal of the Oberwolfach workshop was to bring together researchers representing different aspects of computability theory to discuss recent advances, and to stimulate future work.

This special issue contains 11 carefully selected articles of high calibre that have been reviewed following the usual standards of our discipline. These articles represent a cross section through topics that have been presented and discussed during the workshop. We would like to use this opportunity to thank the authors for their excellent contributions and the additional reviewers for their thorough work.

\section{References}

[1] V. Brattka, R. Downey, J.F. Knight and S. Lempp (eds), Computability theory, Technical report, 1/2018, Mathematisches Forschungsinstitut Oberwolfach, Oberwolfach, Germany, 2018, https: / /www. mfo. de/ document/1802/OWR_2018_01.pdf. 